\title{
A Systematic Review on Trunk Impairment Scale for Stroke Patients
}

\author{
Min Joo Lee ${ }^{a}$, Seul Lee ${ }^{\mathfrak{b}}$, Dae-Sung Park ${ }^{\star \star}(1)$

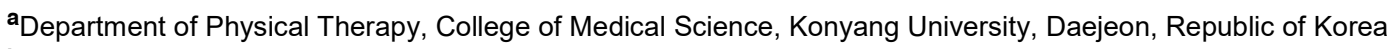 \\ ${ }^{b}$ Department of Physical Therapy, The Graduate School, Konyang University, Daejeon, Republic of Korea
}

Objective: The purpose of this study was to systematically review the trunk impairment scale that are used to assess the trunk control of stroke patients.

Design: A systematic review

Methods: Stroke subjects were categorized as acute, subacute, chronic. In this systematic review, the studies published between 2000 and 2020 were selected. A literature search using the keywords 'QUADAS', 'stroke', 'trunk impairment scale'. Data sources included RISS, GOOGLE Scholar and DBpia. We assessed the quality of assessment tools using Quality Assessment of Diagnostic Accuracy Studies tool.

Results: We reviewed 18 studies. 7 of the 18 studies reported reliability results, 10 reported validity results. The QUADAS tool quality evaluation of 17 literatures extracted except for one randomized control test among 18 literatures showed a range of 3 to 13 points. 5 of the 18 studies are presented with the Cronbach alpha coefficient indicating reliability using internal consistency, all of which are more than 0.8 . All studies that presented test-retest reliability, intra-rater reliability, and inter-rater reliability showed high agreement with an intra-class correlation coefficient of 0.75 or more.

Conclusions: A systematic review of the study of the application of the trunk impairment scale for stroke patients will help provide criteria for future studies and application of the trunk impairment scale in clinical practice.

Key Words: QUADAS, Stroke, Systematic review, Trunk Impairment Scale

서론

뇌졸중은 전 세계적으로 장애와 사망의 주요 원인이다 [1]. 뇌졸중 생존자는 일반적으로 독립적인 삶을 영위할 수 있는 능력에 심각한 영향을 미치는 운동 통제와 균형 이 손상되어 어려움을 겪는다[2]. 이 환자들은 비정상적인 운동과 근긴장도로 인해 자세를 유지, 조절하는 능력이 떨어지고 협응 능력 또한 장애가 나타나 정상적인 체간 조절을 하는 것에 어려움이 발생한다[3]. SF Tyson 등[4] 은 대상자의 뇌졸중 병리, 인구통계, 균형 장애, 기능, 신 경학적 장애에 대한 실험에 참여한 뇌졸중 환자 중 $83 \%$ 가 균형 장애를 갖고 있다고 보고했으며, 이들 중 $27 \%$ 는 앉을 수는 있으나 서는 것은 불가능, $40 \%$ 는 서는 것은 가능하나 보행이 불가능, $33 \%$ 는 보행이 가능하나 균형에
는 제한이 있음을 보였다.

균형에 대한 종합적인 임상 평가는 진단적 이유와 치 료적 이유 모두에 중요하다. 균형 장애는 낙상과 관련한 상해로 이어져 신체 기능에 심각한 결과를 초래할 수 있 다. 또한 사회적 기능에도 영향을 미쳐 사회적 고립으로 이어질 수 있으며, 활동 제한에도 영향을 미치게 된다 [5].

체간의 균형은 뇌졸중 환자의 재활에 매우 중요하다. 체간 조절은 상하지의 움직임에 대해 선행되며, 이런 움 직임에 대한 다른 동작들에 있어 항상 먼저 활성화된다 [6]. 하지만 체간 조절 능력의 회복은 중요하나 그 중요성 이 간과되어 왔으며, 뇌졸중 환자의 체간 회복에 대한 연 구도 비교적 미비하다[7]. 앉은 자세 균형은 뇌졸중 이후 운동 및 기능적 회복의 예측 변수라고 보고되었으며 $[8$,

Received: Aug 23, 2021 Revised: Sep 28, 2021 Accepted: Sep 30, 2021

Corresponding author: Dae-Sung Park (ORCID https://orcid.org/0000-0003-4258-0878)

Department of Physical Therapy, College of Medical Science, Konyang University,158, Gwanjeodong-ro, Seo-gu, Daejeon, Rep. of KOREA [35365]

This is an Open-Access article distributed under the terms of the Creative Commons Attribution Non-Commercial License (http://creativecommons.org/licenses/ by-nc/4.0) which permits unrestricted non-commercial use, distribution, and reproduction in any medium, provided the original work is properly cited.

Copyright @ 2021 Korean Academy of Physical Therapy Rehabilitation Science 
9], 환자의 치료에 있어서 중요한 목표가 된다[10]. 따라 서 체간의 이동성과 안정성을 평가하기 위한 도구의 필요 성이 제기되었다. 그러나 그동안의 뇌졸중 환자의 균형에 대한 대부분의 연구는 기립자세 위주로 진행되었으며, $\mathrm{G}$ Verheyden 등[3]은 뇌졸중 후 운동 재활에 관한 대부분 의 문헌은 상지와 하지에 초점을 맞추고 있으며 체간의 재활은 거의 관심을 받지 못하고 있다고 언급하며 뇌졸중 환자 체간 기능의 측정을 위한 치료적 도구의 필요성을 주장하며 체간장애척도(Trunk Impairment Scale)를 제시 하였다.

체간장애척도는 뇌졸중 환자의 정적 앉은 자세 균형, 동적 앉은 자세 균형, 체간 움직임의 협응력을 평가하기 위해 개발되었다. 체간장애척도는 test-retest agreement (ICC 0.96), Inter-observer agreement(ICC 0.99)에 대한 높은 값을 제시한다[3].

지금까지의 관련 연구들을 살펴보면, 뇌졸중 환자에게 체간장애척도를 적용하여 움직임을 평가하거나 중재를 평 가하기 위한 다양한 연구가 발표되었다 $[11,12,13,14$, $15,16,17]$. 그러나 이러한 연구들은 신뢰도 및 타당도 평가 연구 $[11,12,13]$, 중재에 대한 평가를 위한 도구로 사용된 연구 $[14,15]$, 다른 평가도구들과의 상관관계 평가 연구 $[16,17]$ 등이 주를 이루고 있으며 뇌졸중 환자에 대 한 체간장애척도적용에 대해 전반적으로 평가한 연구는 부족한 상황이다.

이에 본 연구는 뇌졸중 환자에 대한 체간장애척도의 적 용을 체계적으로 검토하여 향후 뇌졸중 환자의 체간의 움 직임 및 균형, 협응 능력에 대한 평가와 재활 치료에 유용 한 기초 자료로 삼고자 한다.

본 연구의 목적은 뇌졸중 환자에 대한 체간장애척도 적 용 연구들을 체계적으로 고찰하는 것으로 구체적인 목적 은 다음과 같다.

첫째, 뇌졸중 환자에 대한 체간장애척도 적용 연구들의 질을 파악한다.

둘째, 향후 연구들과 임상에서의 체간장애척도 적용에 대한 기준을 제공하고자 한다.

\section{연구 방법}

\section{연구 설계}

본 연구는 뇌졸중 환자의 체간장애척도에 관한 체계적 문헌고찰 연구이다.

\section{문헌의 선정기준 및 배제기준}

본 연구에서는 체계적 문헌 고찰을 시행하기 위하여 '체간장애척도가 뇌졸중 환자에게 체간 기능 측정의 신뢰
도와 타당도가 얼마나 되나?'를 핵심질문으로 선정하였다. 구체적 질문과 선정기준 및 배제기준은 다음과 같다.

\section{산정기준}

(1) 뇌졸중 환자를 대상으로 한 연구

(2) 체간장애척도를 평가한 연구

(3) 2000 년 1월 2020년 12월에 출판된 연구

(4) 한글, 영어로 쓰인 연구

(5) 학술지에 게재된 연구

\section{배제기준}

(1) 전문을 구할 수 없는 연구

(2) 한 명의 환자를 대상으로 연구한 사례연구

\section{문헌 검색과 선정}

뇌졸중 관련 체간장애척도의 연구를 정리하고자 2020 년 9월부터 2020년 10월까지 검색을 통하여 2000년부터 2020년까지의 논문을 데이터베이스 한국교육학술정보원 (RISS), GOOGLE Scholar 및 DBpia를 이용해 검색하였 다. 검색어는 'trunk impairment scale', 'stroke'로 검색 하였다. 2 명의 저자가 검색된 논문의 전문을 확인한 후 포함기준과 배제기준에 근거하여 총 18 개의 연구를 선정 하였다(Figure 1).

대상자의 발병 단계는 '7일 $\leq$ 급성기', '7일<아급성기 $\leq 3$ 개월', '만성기 $>3$ 개월'로 설정하였다.

\section{문헌의 질 평가}

선정된 18 편의 논문에 대한 질 평가를 논의하며 시행 하였다. QUADAS(Quality Assessment of studies of Diagnostic Accuracy included Systemic reviews)는 체 계적 문헌고찰 연구에서 진단적 도구개발논문의 질 평가 를 위해 개발된 도구이다. 짧고 간편하며 여러 가지 배경 의 방법론적 연구에 두루 사용할 수 있는 장점이 있다. $\mathrm{QUADAS}$ 는 의학 분야에서 진단적 검사의 질을 평가하 기 위한 목적으로 개발되었다[18].

QUADAS는 체크리스트 형식으로 되어 있으며 문항 수는 14 개로 '그렇다(yes)', '아니다(no)', '불분명하다 (unclear)'의 세 가지로 체크하게 되어 있다. 14 개의 문항 은 “대표성 있는 표본으로 만들어진 도구인가?”, “표집의 선정기준이 명확한가?”, “참조표준이 있는가?”, “참조표준 과 본 도구의 시간차가 적은가?”, “참조표준에 의해 검증 받았는가?”, “동일한 참조표준으로 검증받았는가?”, “본 도구는 참조표준과 독립적인 구성을 가지고 있는가?”, “재현할 수 있을 만큼 세부적인 방법을 제시하는가?”, 


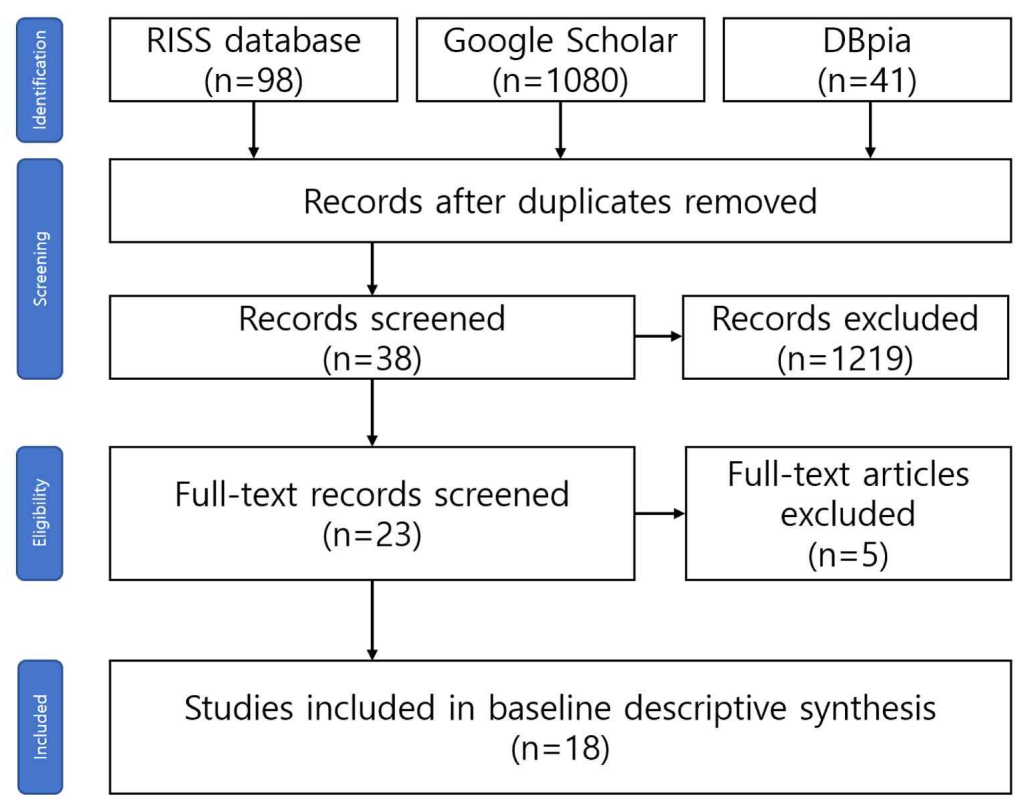

Figure 1. PRISMA flow diagram for systematic review

"반복해 사용할 수 있을 정도로 세부적인 내용을 제시하 는가?”, “개발된 본 도구를 해석할 수 있는가?”, “참조표 준으로 측정한 결과를 해석할 수 있는가?”, “실무에 실행 할 때와 같은 임상적 자료를 나타내는가?”, “중개효과를 가진 측정결과가 보고되었는가?”, “탈락이 설명되었는 가?"의 내용으로 되어 있으며 체크한 점수의 범위는 0-14 점이다. 연구자들은 추출한 문헌들의 질 평가를 하였으며 이에 따른 점수를 확인하였다[19](Table 2).

\section{자료추출과 통합}

최종적으로 선정된 18 편의 논문으로 자료를 추출하였 으며, 포함된 요소로는 연구정보(저자와 출판년도), 대상 자 수, 대상자의 발병 단계 및 유병 기간, 연구의 신뢰도 와 타당도 결과에 대한 자료를 통합하였다. 자료 추출 및 통합은 제 1 저자와 2 저자의 의견과 교신과의 토의과정을 통해 이루어졌다.

\section{결과}

\section{연구 대상자에 관한 일반적인 특성}

모두 840 명의 대상자가 18 편의 연구를 통해 체간장 애척도를 분석하기 위해 모집되었다. 각 연구에서는 15 명에서부터 201명의 대상자가 각 그룹에 포함되었다. 뇌졸중에 의한 급성기와 만성기 환자로 다양하게 나누 어져 연구되었고, 급성기 뇌졸중 환자를 대상으로 한 연 구가 1 편 $(6 \%)$, 아급성기 뇌졸중 환자를 대상으로 한 연
구가 2편(11\%), 만성기 뇌졸중 환자를 대상으로 한 연 구가 5편(28\%), 급성기와 아급성기 뇌졸중 환자를 대상 으로 한 연구가 3 편(17\%), 아급성기와 만성기 뇌졸중 환자를 대상으로 한 연구가 4편(22\%), 급성기, 아급성 기 및 만성기 뇌졸중 환자를 대상으로 한 연구가 3편 $(17 \%)$ 이었다. 연구 대상자들의 연령은 연령이 기재되어 있지 않은 1 편의 연구를 제외하고 50 대에서 60 대가 주 를 이루었다. 연구 대상자에 관한 일반적 특성은 Table 1 과 같다.

\section{체간장애척도에 대한 질 평가}

추출한 문헌 18 편 중 무작위대조시험 연구인 1편을 제 외하고 추출한 17 편의 문헌의 QUADAS 도구개발 문헌 의 질 평가 결과 14 점 만점 중에 3 점에서 13 점의 범위를 나타내었다. 3점으로 평가된 문헌은 Karthikbabu[20]와 $\mathrm{Yu}[21]$ 의 2편이었으며, 13점으로 평가된 문헌은 $\mathrm{S}$. Pathak[22]이었다. 나머지 문헌은 5점인 문헌 3편, 6점인 문헌 2편, 8 점인 문헌 1편, 10 점인 문헌 2편, 11 점인 문 헌 2편, 12 점인 문헌 4편이었다. QUADAS 각 항목별로 실무에 실행했을 때와 같은 임상적 자료를 나타냄은 17 편 이 충족하였다. 반복해 사용할 수 있을 정도로 세부적인 내용을 제시함의 항목은 ‘아니다(No)'가 나타난 문헌이 15 편으로, '아니다(No)'가 가장 많은 항목이었다. 참조표 준과 본 도구의 적은 시간차 항목은 '불분명(Unclear)'이 나타난 문헌이 6편으로, ‘불분명(Unclear)’이 가장 많은 항목이었다(Table 2). 
Table 1. General Characteristics of Subjects $(n=840)$

\begin{tabular}{|c|c|c|c|c|c|}
\hline No. & Author & Year & Stage of stroke (n) & $\begin{array}{l}\text { Onset time }(d / w / m / y) \\
(\text { mean } \pm \text { SD })\end{array}$ & $\begin{array}{l}\text { Age } \\
(\text { mean } \pm \text { SD })\end{array}$ \\
\hline 13 & Näf et al. & 2020 & subacute $(\mathrm{n}=20)$ & $35.00 \pm 23.00 \mathrm{~d}$ & $75.00 \pm 10.00$ \\
\hline 23 & Gjelsvik et al. & 2012 & acute $(n=201)$ & $4.70 \pm 2.20 \mathrm{w}$ & $72.00 \pm 14.00$ \\
\hline 11 & Lombardi et al. & 2017 & acute, subacute $(n=41)$ & $13.10 \pm 8.50 \mathrm{~d}$ & $68.80 \pm 14.20$ \\
\hline 3 & Verheyden et al. & 2004 & subacute, chronic $(\mathrm{n}=28)$ & $61.00 \mathrm{~d}$ & 63.00 \\
\hline 24 & Cabanas-Valdés et al. & 2016 & $\begin{array}{l}\text { acute }(n=10) \\
\text { subacute }(n=38) \\
\text { chronic }(n=10)\end{array}$ & - & $70.60 \pm 15.30$ \\
\hline 25 & Ko et al. & 2015 & $\begin{array}{l}\text { acute, subacute }(n=39) \\
\text { chronic }(n=12)\end{array}$ & - & 57.78 \\
\hline 15 & Park et al. & 2010 & acute, subacute $(n=24)$ & $11.30 \mathrm{~d}$ & 64.30 \\
\hline 22 & Pathak et al., & 2014 & $\begin{array}{l}\text { acute }(n=20) \\
\text { subacute }(n=8) \\
\text { chronic }(n=10)\end{array}$ & - & $59.52 \pm 11.16$ \\
\hline 20 & Karthikbabu et al. & 2011 & chronic $(n=15)$ & $3.53 \pm 2.98 y$ & $50.00 \pm 11.87$ \\
\hline 26 & Ko et al. & 2016 & acute, subacute $(\mathrm{n}=30)$ & $\begin{array}{l}\text { Exp 1: } 12.00 \mathrm{~d} \\
\text { Exp 2: } 8.50 \mathrm{~d} \\
\text { Exp 1+2: } 11.00 \mathrm{~d}\end{array}$ & $\begin{array}{l}\operatorname{Exp~1:~} 59.50 \\
\text { Exp 2: } 65.50 \\
\operatorname{Exp} 1+2: 58.50\end{array}$ \\
\hline 28 & Seo et al. & 2008 & subacute, chronic $(n=25)$ & $1.00 \mathrm{~m} \sim$ over $1.00 \mathrm{y}$ & - \\
\hline 29 & Ha et al. & 2020 & chronic $(n=21)$ & over $6.00 \mathrm{~m}$ & $\begin{array}{l}\text { Exp: } 61.20 \pm 23.79 \\
\text { Control: } 64.91 \pm 16.59\end{array}$ \\
\hline 27 & Verheyden et al. & 2007 & subacute, chronic $(\mathrm{n}=102)$ & $\begin{array}{l}\text { subacute: } 20.00 \mathrm{~d}, \\
\text { chronic: over } 6.00 \mathrm{~m}\end{array}$ & $70.00 \pm 10.00$ \\
\hline 16 & Jijimol et al. & 2013 & chronic $(n=30)$ & $6.00 \sim 36.00 \mathrm{~m}$ & $49.90 \pm 2.80$ \\
\hline 17 & Kim et al. & 2015 & chronic $(n=23)$ & over $6.00 \mathrm{~m}$ & $59.39 \pm 9.22$ \\
\hline 21 & Yu et al. & 2013 & chronic $(n=20)$ & $\begin{array}{l}\text { Exp: } 25.85 \pm 9.99 \mathrm{~m} \\
\text { Control: } 30.96 \pm 7.67 \mathrm{~m}\end{array}$ & $\begin{array}{l}\text { Exp: } 50.00 \pm 5.53 \\
\text { Control: } 52.64 \pm 4.56\end{array}$ \\
\hline 14 & Verheyden et al. & 2009 & subacute $(\mathrm{n}=33)$ & $\begin{array}{l}\text { Exp: } 53.00 \pm 24.00 \mathrm{~d} \\
\text { Control: } 49.00 \pm 28.00 \mathrm{~d}\end{array}$ & $\begin{array}{l}\text { Exp: } 55.00 \pm 11.00 \\
\text { Control: } 62.00 \pm 14.00\end{array}$ \\
\hline 12 & Sag et al. & 2019 & subacute, chronic $(n=80)$ & $1.94 \pm 1.72 \mathrm{~m}$ & $63.00 \pm 12.10$ \\
\hline
\end{tabular}


Table 2. Quality Appraisal of the Studies

\begin{tabular}{|c|c|c|c|c|c|c|c|c|c|c|c|c|c|c|c|c|c|c|}
\hline QUADAS & 13 & 23 & 11 & 3 & 24 & 25 & 15 & 22 & 20 & 26 & 28 & 29 & 27 & 16 & 17 & 21 & 14 & 12 \\
\hline Patient representativeness & Y & $\mathrm{N}$ & Y & $\mathrm{Y}$ & $\mathrm{Y}$ & Y & Y & Y & Y & Y & Y & Y & Y & Y & Y & Y & - & Y \\
\hline Selection criteria & $\mathrm{Y}$ & $\mathrm{Y}$ & $\mathrm{Y}$ & $\mathrm{Y}$ & $\mathrm{Y}$ & $\mathrm{Y}$ & $\mathrm{Y}$ & $\mathrm{Y}$ & $\mathrm{Y}$ & $\mathrm{Y}$ & $\mathrm{N}$ & $\mathrm{Y}$ & $\mathrm{Y}$ & $\mathrm{Y}$ & $\mathrm{Y}$ & $\mathrm{Y}$ & - & $\mathrm{Y}$ \\
\hline Reference standard & $\mathrm{Y}$ & $\mathrm{N}$ & $\mathrm{Y}$ & $\mathrm{Y}$ & $\mathrm{N}$ & $\mathrm{N}$ & $\mathrm{Y}$ & $\mathrm{Y}$ & $\mathrm{N}$ & $\mathrm{Y}$ & $\mathrm{N}$ & $\mathrm{N}$ & Y & $\mathrm{Y}$ & $\mathrm{Y}$ & $\mathrm{N}$ & - & $\mathrm{Y}$ \\
\hline Time period & $\mathrm{Y}$ & $\mathrm{N}$ & $\mathrm{U}$ & $\mathrm{U}$ & $\mathrm{N}$ & $\mathrm{N}$ & $\mathrm{U}$ & $\mathrm{Y}$ & $\mathrm{N}$ & $\mathrm{Y}$ & $\mathrm{N}$ & $\mathrm{N}$ & $\mathrm{U}$ & Y & $\mathrm{U}$ & $\mathrm{N}$ & - & $\mathrm{U}$ \\
\hline verification of reference standard & $\mathrm{Y}$ & $\mathrm{N}$ & $\mathrm{Y}$ & $\mathrm{Y}$ & $\mathrm{N}$ & $\mathrm{N}$ & $\mathrm{Y}$ & $\mathrm{Y}$ & $\mathrm{N}$ & $\mathrm{Y}$ & $\mathrm{N}$ & $\mathrm{N}$ & $\mathrm{Y}$ & $\mathrm{Y}$ & $\mathrm{Y}$ & $\mathrm{N}$ & - & $\mathrm{Y}$ \\
\hline Same reference standard & $\mathrm{Y}$ & $\mathrm{N}$ & $\mathrm{Y}$ & $\mathrm{Y}$ & $\mathrm{N}$ & $\mathrm{N}$ & $\mathrm{Y}$ & Y & $\mathrm{N}$ & Y & $\mathrm{N}$ & $\mathrm{N}$ & Y & Y & Y & $\mathrm{N}$ & - & $\mathrm{Y}$ \\
\hline Independence of index test & $\mathrm{Y}$ & $\mathrm{N}$ & $\mathrm{U}$ & $\mathrm{U}$ & $\mathrm{N}$ & $\mathrm{N}$ & $\mathrm{Y}$ & $\mathrm{Y}$ & $\mathrm{N}$ & $\mathrm{Y}$ & $\mathrm{N}$ & $\mathrm{N}$ & $\mathrm{Y}$ & $\mathrm{Y}$ & $\mathrm{Y}$ & $\mathrm{N}$ & - & $\mathrm{Y}$ \\
\hline Description of reference standard & $\mathrm{N}$ & $\mathrm{Y}$ & Y & Y & $\mathrm{Y}$ & Y & $\mathrm{Y}$ & $\mathrm{N}$ & $\mathrm{N}$ & $\mathrm{N}$ & $\mathrm{N}$ & $\mathrm{N}$ & $\mathrm{N}$ & $\mathrm{N}$ & $\mathrm{N}$ & $\mathrm{N}$ & - & $\mathrm{N}$ \\
\hline Description of index standard & $\mathrm{Y}$ & $\mathrm{N}$ & $\mathrm{N}$ & $\mathrm{N}$ & $\mathrm{N}$ & $\mathrm{N}$ & $\mathrm{N}$ & Y & $\mathrm{N}$ & $\mathrm{N}$ & $\mathrm{N}$ & $\mathrm{N}$ & $\mathrm{N}$ & $\mathrm{N}$ & $\mathrm{N}$ & $\mathrm{N}$ & - & $\mathrm{N}$ \\
\hline Index test results interpretation & $\mathrm{N}$ & $\mathrm{N}$ & $\mathrm{Y}$ & $\mathrm{Y}$ & $\mathrm{N}$ & $\mathrm{N}$ & $\mathrm{Y}$ & Y & $\mathrm{N}$ & Y & Y & $\mathrm{N}$ & Y & Y & Y & $\mathrm{N}$ & - & Y \\
\hline Reference standard results interpretation & $\mathrm{Y}$ & $\mathrm{N}$ & $\mathrm{N}$ & $\mathrm{N}$ & $\mathrm{N}$ & $\mathrm{N}$ & Y & Y & $\mathrm{N}$ & Y & $\mathrm{N}$ & $\mathrm{N}$ & Y & Y & Y & $\mathrm{N}$ & - & $\mathrm{N}$ \\
\hline Clinical availability & Y & $\mathrm{Y}$ & Y & Y & Y & Y & $\mathrm{Y}$ & Y & Y & Y & Y & Y & Y & Y & Y & Y & - & Y \\
\hline Intermediate test result & Y & Y & Y & $\mathrm{U}$ & $\mathrm{Y}$ & Y & Y & Y & $\mathrm{N}$ & Y & $\mathrm{Y}$ & Y & Y & $\mathrm{Y}$ & $\mathrm{Y}$ & $\mathrm{N}$ & - & Y \\
\hline Explanation of withdrawal & $\mathrm{Y}$ & $\mathrm{Y}$ & $\mathrm{Y}$ & $\mathrm{U}$ & $\mathrm{Y}$ & $\mathrm{Y}$ & $\mathrm{Y}$ & $\mathrm{Y}$ & $\mathrm{N}$ & $\mathrm{Y}$ & $\mathrm{Y}$ & $\mathrm{Y}$ & Y & $\mathrm{Y}$ & Y & $\mathrm{N}$ & - & $\mathrm{Y}$ \\
\hline Total score & 12 & 5 & 10 & 8 & 6 & 6 & 12 & 13 & 3 & 12 & 5 & 5 & 11 & 12 & 11 & 3 & & 10 \\
\hline
\end{tabular}

QUADAS: Quality Assessment of studies of Diagnostic Accuracy included Systemic reviews,

Y: Yes, N: No, U: Unclear.

\section{체간장애척도를 보고한 연구의 특성}

본 연구에서 고찰한 18 편의 연구의 특성은 연구자, 게 재년도, 연구 국가, 신뢰도 결과, 타당도 결과로 분류하 였다. 게재년도 별로는 2000 2005년에 보고된 연구가 1편(6\%)이었으며, 2006 2010년에 보고된 연구는 4 편(22\%), 2011 2015년에 보고된 연구는 7편(39\%), 2016 2020년에 보고된 연구는 6편(33\%)이었다. 연구 국가는 아시아 10 편(56\%), 유럽 7편(39\%), 기타 1편 (6\%) 순이었다.

18 편의 연구 중에서 내적일관성을 이용한 신뢰성을 나 타내는 크론바흐 알파계수를 제시한 경우는 5 편 $[3,11$, $12,23,24]$ 이며 모든 값이 0.8 이상으로 신뢰도가 높은 것으로 나타났다. 검사-재검사 신뢰도(test-retest reliability) 방법에 의한 신뢰성을 제시한 경우는 4 편 $[3,12,25$, 26], 측정자 내 신뢰도(intra-rater reliability) 방법에 의한 신뢰성을 제시한 경우는 3 편 $[11,12,24]$, 측정자 간 신뢰도(inter-rater reliability) 방법에 의한 신뢰성을 제시한 경우는 6 편 $[3,11,12,24,25,26]$ 이었다. 검사 -재검사 신뢰도, 측정자 내 신뢰도 및 측정자 간 신뢰도 를 제시한 연구 모두 급내 상관계수(ICC; intraclass correlation coefficient)가 0.75 이상으로 좋은 일치도를 나타냈다.
18 편의 연구 중에서 타당도를 보고한 연구는 10 편[3, $11,12,13,15,16,17,22,26,27]$ 이었다. Barthel Index (BI)와의 상관 관계를 분석한 연구가 4편 $[3,11$, $12,27]$, Trunk Control Test (TCT)와의 상관 관계를 분 석한 연구가 2편[3, 11], Korean version of modified Barthel index(K-MBI)와의 상관 관계를 분석한 연구가 2 편 $[15,26]$, 그 외 평가와의 상관 관계를 분석한 연구는 각 1 편[13, 16, 17, 22]순이었다. 상관계수에 의한 타당도 평가에서 8 개의 평가가 높은 타당도를 나타냈고, 8 개의 평가가 매우 높은 타당도를 나타냈다(Table 3).

\section{고찰}

뇌졸중 환자에서의 체간 균형은 낙상으로도 이어질 수 있는 큰 문제이다. 낙상은 신체 기능과 활동 제한으로 이 어질 수 있어 사회적 기능에 영향을 미친다. 따라서 체간 의 조절을 통한 이동성과 안정성을 평가하는 것은 향후 운동이나 기능적 회복의 지표가 된다는 점에서 중요하게 여겨진다.

이에 본 연구에서는 뇌졸중 환자에 대한 체간장애척도 적용 연구의 질을 파악하고, 향후 연구들과 임상에서의 체간장애척도 적용에 대한 기준을 제공하기 위해 수행된 


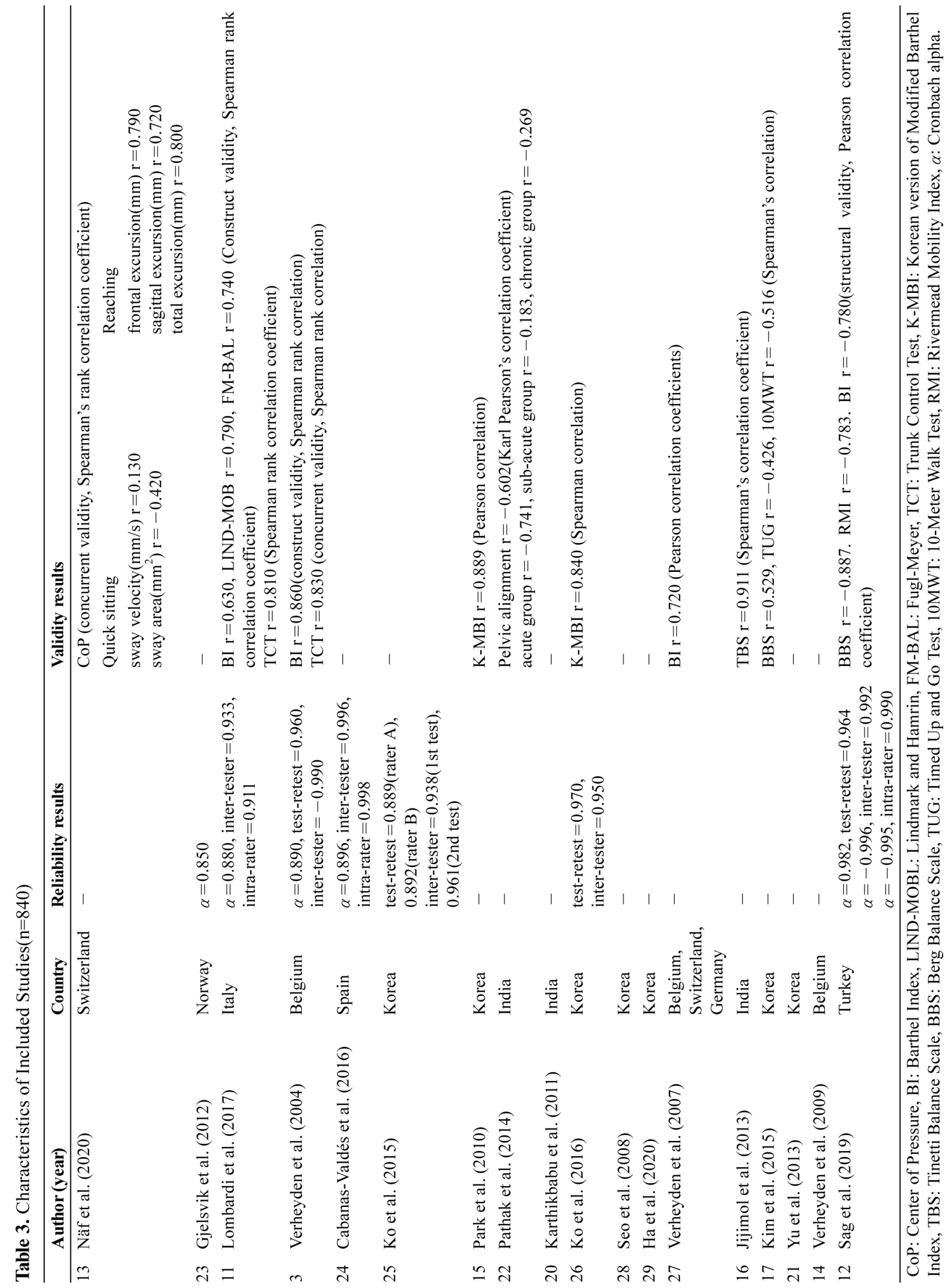


체계적 고찰 연구라는 의의가 있다. 문헌 검색 및 선정 결 과 국내 논문 7 편, 국외 논문 11 편으로 총 18 편이 최종 분석에 포함되었다. 대상자의 발병 단계에 따라서 급성기 대상 연구 1 편, 아급성기 대상 연구 2 편, 만성기 대상 연 구 5편, 급성기와 아급성기 대상 연구 3 편, 아급성기와 만 성기 대상 연구 4편, 급성기와 아급성기 및 만성기 대상 연구 3 편이 분석되었다. 무작위대조시험 연구인 1 편을 제 외한 17 편의 문헌은 QUADAS를 통해 문헌에 대한 질 평가를 실시한 결과 14 점 만점 중에 2편의 3점 평가부터 1 편의 13 점 평가 결과를 나타내었다. 체간장애척도는 뇌 졸중 환자의 예후를 고려할 때 재활 환경에서 예측 도구 로 고려되어 왔다[27]. 내적일관성을 이용한 신뢰성을 나 타내는 크론바흐 알파계수를 제시한 5 편의 연구는 모두 0.8 이상으로 신뢰도가 높은 것으로 나타났다. 18 편의 연 구 중에서 타당도를 제시한 10 편의 연구는 모두 높은 타 당도를 보였다.

본 연구는 뇌졸중 환자의 체간장애척도 적용을 체계적 으로 고찰함에 있어 제한점은 다음과 같다. 첫째, QUADAS 평가 결과 반복해 사용할 수 있을 정도로 체간 장애척도 평가 진행 방법에 대한 세부적인 내용을 제시한 문헌이 18 편 중 3 편에 불과하였다. 둘째, 참조표준과 본 도구의 정확한 시간차를 제시한 문헌이 18 편 중 4 편에 불 과하였으며, 나머지 14편은 불분명하거나(Unclear) 제시 되지 않았다. 이러한 제한점이 있음에도 불구하고 향후 운동이나 기능적 회복의 지표가 되는 체간의 조절에 대한 체간장애척도 적용 연구의 질을 평가하고, 적용 기준을 제공하기 위한 바탕을 제공할 수 있다는 점에 본 연구의 의의가 있다.

\section{결론}

본 연구는 체계적인 문헌 고찰을 통해 뇌졸중 환자에게 시행한 체간장애척도 연구를 찾고, 그 신뢰도와 타당도를 분석하였다. 연구결과 18 편의 연구가 최종적으로 분석되 었다.

그 결과 내적일관성을 이용한 신뢰성을 나타내는 크론 바흐 알파계수를 제시한 연구는 모든 값이 0.8 이상으로 신뢰도가 높았다. 검사-재검사 신뢰도, 측정자 내 신뢰도 및 측정자 간 신뢰도를 제시한 연구 모두 급내 상관계수 가 0.75 이상으로 좋은 일치도를 나타냈다. 타당도 평가 는 8 개의 평가가 높은 타당도를 나타냈고, 8 개의 평가가 매우 높은 타당도를 나타냈다.

이를 통해 뇌졸중 환자에 대한 체간장애척도 적용이 기 립자세 위주의 균형 평가에서 벗어나 뇌졸중 환자의 앉은 자세에서의 체간 움직임, 균형, 협응 능력에 대한 평가와 치료의 기준이 될 것이라고 기대된다.

\section{참고문헌}

1. KATAN, Mira; LUFT, Andreas. Global burden of stroke. In: Seminneurol. GeorgThiemeVerlag. 2018; 38.2:208-211.

2. Hariharasudhan, Ravichandran, Janakiraman Balamurugan. Enhancing trunk stability in acute poststroke subjects using physioball exercise and proprioceptive neuromuscular facilitation technique: A pilot randomized controlled trial. IntJAdvMedHealthRes. 2016;3.1: 5-10.

3. Verheyden G., Nieuwboer A., Mertin J., Preger R., Kiekens C., et al. The Trunk Impairment Scale: a new tool to measure motor impairment of the trunk after stroke. Clinehabil.2004;18.3:326-334.

4. Tyson S. F., Hanley M., Chillala J., Selley A., Tallis R. C. Balance disability after stroke. Physther. 2006;86.1:30-38.

5. MANCINI, Martina; HORAK, Fay B. The relevance of clinical balance assessment tools to differentiate balance deficits. EurJPhysRehabilMed. 2010; 46.2:239-248.

6. Verheyden G., Nieuwboer A., Feys H., Thijs V., Vaes K., et al. Discriminant ability of the Trunk Impairment Scale: a comparison between stroke patients and healthy individuals. DisabilRehabil. 2005;27.17:1023-1028.

7. Lanzetta D., Cattaneo D., Pellegatta D., Cardini R. Trunk control in unstable sitting posture during functional activities in healthy subjects and patients with multiple sclerosis. ArchPhysMedRehabil. 2004; 85.2:279-283.

8. SANDIN, Karl J.; SMITH, Barry S. The measure of balance in sitting in stroke rehabilitation prognosis. Stroke. 1990;21.1:82-86.

9. LOEWEN, Sandy C.; ANDERSON, Brian A. Predictors of stroke outcome using objective measurement scales. Stroke. 1990;21.1:78-81.

10. Harley C., Boyd J. E., Cockburn J., Collin C., Haggard P., et al. Disruption of sitting balance after stroke: influence of spoken output. JNeurolNeurosurg Psychiatry. 2006;77.5:674-676.

11. Lombardi B., Orioli A., Casavola D., Paci M. The Italian version of the Trunk Impairment Scale: development and psychometric properties. EurJPhys RehabilMed. 2017;53.4:516-520. 
12. Sag S., Buyukavci R., Sahin F., Sag M. S., Dogu B., et al. Assessing the validity and reliability of the Turkish version of the Trunk Impairment Scale in stroke patients. NorthClinIstanb. 2019;6.2:156-165.

13. Näf O. B., Bauer C. M., Zange C., Rast F. M. Validity and variability of center of pressure measures to quantify trunk control in stroke patients during quiet sitting and reaching tasks. GaitPosture. 2020;76:218-223.

14. Verheyden G., Vereeck L., Truijen S., Troch M., LaFosse C., et al. Additional exercises improve trunk performance after stroke: a pilot randomized controlled trial. NeurorehabilNeuralRepair. 2009;23.3:281-286.

15. Park J. Y., Chun M. H., Kim Y. M., Kang S. H. Trunk Impairment Scale for Evaluation of Functional Improvement in Acute Stroke Patients. JKorAcad RehabilMed. 2010;34.3:278-284.

16. JIJIMOL G.; FAYAZ R. K.; VIJESH P. V. Correlation of trunk impairment with balance in patients with chronic stroke. NeuroRehabilitation. 2013;32.2:323-325.

17. KIM Jung-Hyun; LEE Suk-Min; JEON Seo-Hyun. Correlations among trunk impairment, functional performance, and muscle activity during forward reaching tasks in patients with chronic stroke. JPhysTherSci. 2015;27.9:2955-2958.

18. Whiting P. F., Rutjes A. W., Westwood M. E., Mallett S., Deeks J. J., et al. QUADAS-2: a revised tool for the quality assessment of diagnostic accuracy studies. AnnInternMed. 2011;155.8:529-536.

19. Whiting P., Rutjes A. W., Reitsma J. B., Bossuyt P. M., Kleijnen J. The development of QUADAS: a tool for the quality assessment of studies of diagnostic accuracy included in systematic reviews. BMCMedResMethodol. 2003;3.1:1-13.

20. Karthikbabu S., John M S., Manikandan N., Bhamini K R., Chakrapani M., et al. Role of trunk rehabilitation on trunk control, balance and gait in patients with chronic stroke: a pre-post design. NeurosciMed. 2011;2:61-67

21. YU Seong-Hun; PARK Seong-Doo. The effects of core stability strength exercise on muscle activity and trunk impairment scale in stroke patients. JExercRehabil. 2013;9.3:362-367.

22. Pathak S., Nayak A., Kedambadi R. C. The relationship between pelvic alignment and trunk control in stroke subjects: a cross-sectional study. IntJResMedSci.
2014;2.4:1483-1487.

23. Gjelsvik B., Breivik K., Verheyden G., Smedal T., Hofstad H., et al. The Trunk Impairment Scalemodified to ordinal scales in the Norwegian version. Disabil Rehabil. 2012;34.16:1385-1395.

24. Cabanas-Valdés R., Urrútia G., Bagur-Calafat C., Caballero-Gómez F. M., Germán-Romero A., et al. Validation of the Spanish version of the Trunk Impairment Scale Version 2.0 (TIS 2.0) to assess dynamic sitting balance and coordination in poststroke adult patients. TopstrokeRehab. 2016;23.4:225-232.

25. KO Jooyeon; YOU Youngyoul. Reliability and responsiveness of the Korean version of the trunk impairment scale for stroke patients. JKorPhysTher. 2015;27.4:175-182.

26. Ko E. J., Chun M. H., Kim D. Y., Yi J. H., Kim W., et al. The additive effects of core muscle strengthening and trunk NMES on trunk balance in stroke patients. AnnRehabilMed. 2016;40.1:142-151.

27. Verheyden G., Nieuwboer A., De Wit L., Feys H., Schuback B., et al. Trunk performance after stroke: an eye catching predictor of functional outcome. JNeurolNeurosurgPsychiatry. 2007;78.7:694-698.

28. SEO Hyun-Du; KIM Nam-Joe; CHUNG Yi-Jung. Reliability of the Korean version of the trunk impairment scale in patients with stroke. PhysTherKor. 2008;15.4:87-96.

29. HA Sun-Young; SUNG Yun-Hee. Attentional concentration during physiotherapeutic intervention improves gait and trunk control in patients with stroke. NeurosciLett. 2020,736:135291. 